\title{
Emergence of multi-acaricide resistant Rhipicephalus ticks and its implication on chemical tick control in Uganda
}

Patrick Vudriko ${ }^{1,2,3}$, James Okwee-Acai ${ }^{1}$, Dickson Stuart Tayebwa ${ }^{2}$, Joseph Byaruhanga ${ }^{2}$, Steven Kakooza ${ }^{2}$, Edward Wampande ${ }^{1,2}$, Robert Omara ${ }^{1}$, Jeanne Bukeka Muhindo ${ }^{1,4}$, Robert Tweyongyere ${ }^{1,2}$, David Okello Owiny ${ }^{1,5}$, Takeshi Hatta ${ }^{6,7}$, Naotoshi Tsuji ${ }^{6,7}$, Rika Umemiya-Shirafuji ${ }^{3}$, Xuenan Xuan ${ }^{3}$, Masaharu Kanameda ${ }^{2,8}$, Kozo Fujisaki ${ }^{6}$ and Hiroshi Suzuki ${ }^{3^{*}}$

\begin{abstract}
Background: Acaricide failure has been on the rise in the western and central cattle corridor of Uganda. In this study, we identified the tick species associated with acaricide failure and determined their susceptibility to various acaricide molecules used for tick control in Uganda.

Methods: In this cross sectional study, tick samples were collected and identified to species level from 54 purposively selected farms (from 17 districts) that mostly had a history of acaricide failure. Larval packet test was used to screen 31 tick populations from 30 farms for susceptibility at discriminating dose (DD) and $2 \times$ DD of five panels of commercial acaricide molecules belonging to the following classes; amidine, synthetic pyrethroid (SP), organophosphate (OP) and OP-SP co-formulations (COF). Resistance was assessed based on World Health Organization criteria for screening insecticide resistance.
\end{abstract}

Results: Of the 1357 ticks identified, Rhipicephalus (Rhipicephalus) appendiculatus and Rhipicephalus (Boophilus) decoloratus were the major (95.6\%) tick species in farms sampled. Resistance against SP was detected in $90.0 \%$ (27/30) of the tick populations tested. Worryingly, $60.0 \%(18 / 30)$ and $63.0 \%(19 / 30)$ of the above ticks were super resistant ( $0 \%$ mortality) against $2 \times D D$ cypermethrin and deltamethrin, respectively. Resistance was also detected against COF (43.3\%), OP chlorfenvinphos (13.3\%) and amitraz (12.9\%). In two years, $74.1 \%$ (20/27) of the farms had used two to three acaricide molecules, and $55.6 \%$ (15/27) rotated the molecules wrongly. Multi-acaricide resistance (at least 2 molecules) was detected in $55.2 \%$ (16/29) of the resistant Rhipicephalus ticks and significantly associated with $R$. decoloratus ( $p=0.0133)$, use of both SP and COF in the last 2 years $(p<0.001)$ and Kiruhura district $(p=0.0339)$. Despite emergence of amitraz resistance in the greater Bushenyi area, it was the most efficacious molecule against SP and COF resistant ticks.

Conclusion: This study is the first to report emergence of super SP resistant and multi-acaricide resistant Rhipicephalus ticks in Uganda. Amitraz was the best acaricide against SP and COF resistant ticks. However, in the absence of technical interventions, farmer-led solutions aimed at troubleshooting for efficacy of multitude of acaricides at their disposal are expected to potentially cause negative collateral effects on future chemical tick control options, animal welfare and public health.

Keywords: Ticks, Rhipicephalus appendiculatus, Rhipicephalus (Boophilus) decoloratus, Acaricide, Resistance, Amitraz, Synthetic pyrethroids, Organophosphates

\footnotetext{
* Correspondence: hisuzuki@obihiro.ac.jp

${ }^{3}$ National Research Center for Protozoan Diseases, Obihiro University of

Agriculture and Veterinary Medicine, Inada-Cho, Obihiro, Hokkaido 080-8555,

Japan

Full list of author information is available at the end of the article
} 


\section{Background}

Ticks are one of the leading vectors of diseases of economic importance to the livestock industry in Africa [1-3]. Tickborne diseases (TBD) mainly theileriosis/East Coast fever (ECF), babesiosis and anaplasmosis present serious constraints to productivity of especially exotic cattle and their crosses. In Uganda, over 30 \% of calf crop is lost to TBD [4]. The above diseases also account for nearly $90 \%$ of total disease control costs and over $60 \%$ of total farm inputs [5]. To address tick challenge, commercial cattle farmers rely extensively on acaricides for chemical control of ticks. This has created a huge demand and market for acaricides in Uganda. The liberalization of the veterinary drug industry in the country has made acaricides even more accessible to farmers [6]. Because of limited control, cases of irrational use of acaricides by farmers have been widely reported [6-8]. Wrong dilution, application methods and increased acaricide pressure are amongst factors that accelerate development of acaricide resistance $[9,10]$. Acaricide resistance was first reported in Uganda in 1970 against organochlorine toxaphene by Rhipicephalus (Boophilus) decoloratus and Rhipicephalus evertsi [11]. The lack of tick acaricide resistance monitoring system since early 1990's to date implies that the performance of various molecules on the Ugandan market are unknown. However, the increased cases of farmers' complaints on acaricide failure, especially in western and central cattle corridors, raises serious suspicion of possible emergence of acaricide resistant ticks in the country. In the rest of the world, tick resistance to various classes of acaricides has been extensively reported [10]. Most of the studies reported acaricide resistance against one class of molecules. However, cases of multiple acaricide resistance by Rhipicephalus (Boophilus) microplus have been reported in Mexico [12]. Several methods have been proposed for detection of acaricide resistance. Larval packet test (LPT), larval tarsal test (LTT) and adult immersion test (AIT) are among the common tests used [13-16]. However, the limitations associated with the above tests such as high labour and time requirements have resulted in the introduction of genomic based approaches [16-19]. Nevertheless, the high costs of genomic approaches leaves LPT as the most used tool for routine acaricide resistance screening. This is further consolidated by the greater agreement between LPT and genomic tools $[16,20]$. The current study established the common species of ticks associated with acaricide failure, acaricide use practices and determined the acaricide resistance profile of the ticks using LPT.

\section{Methods}

\section{Study area}

The primary study area for this research were cattle farms in western and central Uganda that were experiencing acaricide failure between December 2013 and January 2015. Western and central Uganda have the highest population of exotic cattle (especially dairy breeds) and their crosses [21]. Due to the susceptibility of the improved breeds, farmers have to rely on extensive use of acaricides for tick control and prevention of TBD. A total of 14 districts from central and western Uganda were included in this study. They were identified during an earlier investigation of complaints of acaricide failure by the National Drug Authority of Uganda and our research team. Thus, the farms from central (16 farms) and western ( 34 farms) Uganda were purposively sampled based on history of acaricide failure reported to the respective district veterinary office and animal health workers. However, 4 additional samples were obtained from 1 district in the north (Gulu) and 2 districts in the eastern (Serere and Mbale) parts of Uganda. The sample from Gulu was collected from cattle in the abattoir to establish possible spread of resistant ticks through cattle trade. The tick samples from Mbale were collected electively for purposes of finding a reference susceptible tick. Overall ticks were collected from 54 study sites designated as farms in this study (Additional file 1: Figure S1).

\section{Tick collection}

Ticks were collected from 6 to 20 randomly sampled cattle per farm although dogs were also included for tick collection in farms that had dogs. Ticks were collected from goats and sheep on one farm in Kampala. Cattle, goats and sheep were restrained and inspected for ticks in the various predilection sites. Dogs on the other hand were restrained by the owner before tick samples were picked. Both engorged and semi-engorged ticks from each farm were carefully picked and put in perforated labelled sample bottles and transported to the Central Diagnostic laboratory (CDL) at the College of Veterinary Medicine, Animal Resources and Biosecurity (COVAB), Makerere University for taxonomic identification, hatching and acaricide efficacy assays.

\section{Taxonomic identification of tick samples}

Ticks were identified to species level based on morphological features described by Walker et al. [22]. For each farm, identified ticks were categorized based on their species to determine the dominant species associated with acaricide failure at farm and district levels. The engorged female ticks were immediately transferred into individual tubes and incubated at $27 \pm 1{ }^{\circ} \mathrm{C}$ and $80 \%$ relative humidity for oviposition. After hatching, the larvae were kept in the incubator until they were 18 days old and used for acaricide efficacy assays. 


\section{Acaricides used for tick resistance assay}

Commercial acaricide formulations that represented all the classes of acaricide on Ugandan market were purchased from the local importers and used for LPT. They were coded as; A4 (12.5\% amitraz, Kenya), SP3 (10\%, $\alpha$-cypermethrin, Kenya), SP10 (5 \%, deltamethrin, Tunisia), OP (100\%, chlorfenvinphos, Italy), COF1 (co-formulation, $30 \%$ chlorfenvinphos and $3 \% \alpha-$ cypermethrin, Italy). The commercial (brand) names of the acaricides used were coded for anonymity to avoid any misinterpretation as promotion or demotion of such products based on their efficacy result.

\section{Tick bioassays for acaricide efficacy}

A total of 31 tick populations from 30 farms were tested for acaricide susceptibility. For logistical reasons, we adopted the method proposed for insecticide resistance testing by World Health Organization (WHO) [23]. The manufacturers recommended concentration was considered as the diagnostic/discriminating dose (DD) for all the chemicals. However, one additional dose level, which was twice the above dose $(2 \times \mathrm{DD})$ was also applied. The diluent used for all the acaricides was trichloroethylene and olive oil mixed in a ratio of 2:1 [24]. For amitraz, the method by Miller et al. [25] was used. Briefly, $0.25 \mathrm{mg} / \mathrm{ml}(\mathrm{DD})$ and $0.5 \mathrm{mg} / \mathrm{ml}(2 \times \mathrm{DD})$ commercial amitraz were prepared using the diluent. For cypermethrin and deltamethrin, $0.05 \mathrm{mg} / \mathrm{ml}$ (DD) and $0.1 \mathrm{mg} / \mathrm{ml}(2 \times$ DD) respectively were prepared. For OP-chlorfenvinphos $0.5 \mathrm{mg} / \mathrm{ml}(\mathrm{DD})$ and $1 \mathrm{mg} / \mathrm{ml}(2 \times \mathrm{DD})$ were prepared for the bioassays. The concentration of the coformulation prepared were 0.3:0.03 $\mathrm{mg} / \mathrm{ml}(\mathrm{DD})$ and 0.6:0.06 $\mathrm{mg} / \mathrm{ml}$ $(2 \times \mathrm{DD})$.

The choice of substrate used for impregnation of the chemicals was based on Food and Agriculture Organization (FAO) [24] recommendation. Filter paper (Whatman No.1, Whatman, Madstone, United Kingdom) was used as a substrate for cypermethrin, deltamethrin, chlorfenvinphos and co-formulated acaricide. Nylon fabric was used for amitraz. The substrates were labelled with pencil and impregnated with $0.7 \mathrm{ml}$ of the corresponding acaricide solution prepared. Trichloroethylene was evaporated in a fume hood for 2 hours. Each impregnated filter paper or nylon fabric was folded into a packet and loaded with, on average, 60 larvae from the same farm and same species. The packets were then secured with alligator clips and incubated at $29 \pm$ $1{ }^{\circ} \mathrm{C}$ and $80 \%$ relative humidity for 24 hours. Each experiment was carried out in duplicate. In all the assays, contamination was avoided by starting every experiment with the negative control followed by the lower concentration and changing gloves between different acaricide molecules. In the absence of laboratory reference susceptible Rhipicephalus ticks in the country, Haemaphysalis leachi and Amblyoma variegatum larvae that were $100 \%$ susceptible to all the acaricides were taken as reference ticks for phenotypic acaricide resistance assay. The reliability of this approach was later verified using 6 populations of susceptible $R$. appendiculatus and $R$. decoloratus reference ticks collected from low acaricide pressure farms in Adjumani district-north western Uganda (Unpublished data).

After 24 hours, the packets were removed in the order in which they were loaded in the incubator. Three independent enumerators who were previously trained on identifying dead and live ticks using a magnifying lens and stereo-microscope counted the number of ticks that died and those that were alive for each set of experiments. Mortalities were expressed as percentage of the total number of larvae exposed to the acaricide. There were no mortalities recorded in the control groups that were exposed to only the diluent.

\section{Data on acaricide application practices}

A semi-structured interview with farmers and/or farm workers was carried out from 52 of the 54 farms since data could not be retrieved from the two farms. The data captured included breeds of cattle reared, sequence of acaricide brands used in the last two years, method of acaricide application, dilution of acaricide(s) used, application interval at the time of the study and mixing of two or more acaricide formulations at one time. The data on sequence and brands of acaricides were used to determine the correctness of rotation from one molecule to another. Rotation was considered wrong if a farmer changed acaricide brand within the same molecule and changing from COF to SP following acaricide failure. However, a change from synthetic pyrethroid (SP) to co-formulation (COF) and organophosphate (OP) molecule following acaricide failure to SP was also considered a wrong rotation due to the possible crossresistance between SP and OP [10, 26, 27]. The farm data on acaricide usage was also used to establish the brand preference for the different acaricide molecules on the market. The registration status of various brands of acaricides stated by the farmers was either established from National Drug Authority or verified using the National Drug Authority's Veterinary Register (http:// www.nda.or.ug/docs/Vet_List.pdf).

\section{Data analysis}

The mortality data for the 31 tick populations tested were recorded in MS excel and mean mortality and standard error determined. The WHO [23] percentage mortality cut-off values for susceptibility and resistance against insecticides determined using DD were used to categorize the mortality data. Ticks that showed at least $80 \%$ mortality were considered susceptible while those that showed less than $80 \%$ mortality against a given chemical were considered resistant. The above data 
together with the qualitative data on acaricide use was analysed using SPSS version 21 (IBM SPSS Statistics for Windows, Version 21.0. Armonk, NY: IBM Corp.). Pearson chi square analysis was done with MedCalc for Windows, version 12.5 (MedCalc Software, Ostend, Belgium) to determine the factors associated with multiple acaricide resistance at $95 \%$ confidence and $p$ value $\leq 0.05$ was considered statistically significant.

\section{Ethical considerations}

The study was approved by the institutional review board (No. VAB/REC/15/104) of the College of Veterinary Medicine, Animal Resources and Biosecurity, Makerere University. To ensure biosecurity of ticks, all experiments were carried out under strict in-house procedure for avoiding escape of larvae. All materials used were either autoclaved or soaked in hot water at $99{ }^{\circ} \mathrm{C}$. Larvae that were kept for further molecular studies were preserved in $70 \%$ ethanol. The commercial (brand) names of all the acaricides were coded to ensure confidentiality.

\section{Results}

\section{Farm characteristics and tick species identified}

Of the 54 cattle farms from which ticks were collected, $83.3 \%$ (45/54) kept crosses of exotic cattle and 9/54 had only local cattle as the main livestock enterprise. Up to $90.4 \%(47 / 52)$ of the farms used hand spray for acaricide application while only $3.8 \%(2 / 52)$ used plunge dip and another $1.9 \%(1 / 52)$ used spray race. Complaint of acaricide failure was reported in $94.4 \%(51 / 54)$ of the farms that were all located in central and western Uganda. A total of 1357 ticks were identified from the 54 study farms. Rhipicephalus ticks accounted for $95.6 \%$ (1297/1357) of the tick populations although A.variegatum and H. leachi constituted $3.5 \%$ (48/1357) and $0.9 \%$ (12/1357), respectively. Amongst the Rhipicephalus, $55.1 \%(715 / 1297)$ were the one host ticks $R$. decoloratus compared to $44.9 \%(582 / 1297)$ three host tick Rhipicephalus appendiculatus. On the other hand, $70.8 \%$ (34/ 48 ) of the A. variegatum ticks were from eastern Uganda (Table 1). Only one out of the $12 \mathrm{H}$. leachi was collected on cattle, the rest were from dogs. No Rhipicephalus tick was found on dogs. For the 51 farms that had complaints of acaricide failure, $98.0 \%(1257 / 1283)$ of the ticks belonged to the genus Rhipicephalus. Rhipicephalus (Boophilus) decolortus were $55.7 \%(714 / 1257)$ and $42.3 \%$ (543/1257) were $R$. appendiculatus. A. variegatum formed only $1.1 \%(14 / 1257)$ of the ticks from the 51 farms.

\section{Acaricide molecules and brand preferences by farmers}

The veterinary drug register showed that a total of 25 commercial brands of acaricides had been marketed in Uganda. Synthetic pyrethroids (SP1-SP15) constituted

Table 1 Species of ticks identified from the various study areas

\begin{tabular}{|c|c|c|c|c|c|c|c|c|c|c|c|}
\hline \multirow[b]{3}{*}{ Region } & \multirow[b]{3}{*}{ District } & \multirow[b]{3}{*}{ No.farms } & \multicolumn{7}{|c|}{ Number and frequency (\%) per district } & \multirow[b]{3}{*}{$\%$} & \multirow[b]{3}{*}{ Total } \\
\hline & & & \multicolumn{2}{|c|}{ R. appendiculatus } & \multicolumn{2}{|c|}{ R. decoloratus } & \multicolumn{2}{|c|}{ A. variegatum } & \multirow{2}{*}{$\begin{array}{l}\text { H. leachi } \\
\text { No. }\end{array}$} & & \\
\hline & & & No. & $\%$ & No. & $\%$ & No. & $\%$ & & & \\
\hline \multirow[t]{9}{*}{ Central } & Kampala & 1 & 17 & 100.0 & 0 & 0.0 & 0 & 0.0 & 0 & 0.0 & 17 \\
\hline & Kiboga & 1 & 6 & 60.0 & 4 & 40.0 & 0 & 0.0 & 0 & 0.0 & 10 \\
\hline & Kyankwanzi & 1 & 1 & 9.1 & 4 & 36.4 & 2 & 18.2 & 4 & 36.4 & 11 \\
\hline & Mpigi & 1 & 30 & 100.0 & 0 & 0.0 & 0 & 0.0 & 0 & 0.0 & 30 \\
\hline & Mubende & 1 & 0 & 0.0 & 35 & 100.0 & 0 & 0.0 & 0 & 0.0 & 35 \\
\hline & Nakasongola & 1 & 15 & 48.4 & 9 & 29.0 & 7 & 22.6 & 0 & 0.0 & 31 \\
\hline & Sembabule & 7 & 79 & 73.1 & 29 & 26.9 & 0 & 0.0 & 0 & 0.0 & 108 \\
\hline & Wakiso & 3 & 31 & 52.5 & 28 & 47.5 & 0 & 0.0 & 0 & 0.0 & 59 \\
\hline & Mbale & 2 & 1 & 2.9 & 1 & 2.9 & 33 & 94.3 & 0 & 0.0 & 35 \\
\hline East & Serere & 1 & 0 & 0.0 & 13 & 92.9 & 1 & 7.1 & 0 & 0.0 & 14 \\
\hline North & Gulu & 1 & 38 & 97.4 & 0 & 0.0 & 1 & 2.6 & 0 & 0.0 & 39 \\
\hline \multirow[t]{6}{*}{ West } & Bushenyi & 4 & 3 & 0.9 & 347 & 99.1 & 0 & 0.0 & 0 & 0.0 & 350 \\
\hline & Kiruhura & 12 & 28 & 17.8 & 121 & 77.1 & 0 & 0.0 & 8 & 5.1 & 157 \\
\hline & Mbarara & 6 & 96 & 61.5 & 56 & 35.9 & 4 & 2.6 & 0 & 0.0 & 156 \\
\hline & Mitoma & 3 & 20 & 29.4 & 48 & 70.6 & 0 & 0.0 & 0 & 0.0 & 68 \\
\hline & Rukungiri & 8 & 217 & 95.6 & 10 & 4.4 & 0 & 0.0 & 0 & 0.0 & 227 \\
\hline & Sheema & 1 & 0 & 0.0 & 10 & 100.0 & 0 & 0.0 & 0 & 0.0 & 10 \\
\hline Total & 17 & 54 & 582 & 42.9 & 715 & 52.7 & 48 & 3.5 & 12 & 0.9 & 1357 \\
\hline
\end{tabular}


$60.0 \%(15 / 25)$ of the total commercial brands marketed, followed by amitraz (A1-A7 brands) $28.0 \%$ (7/25), coformulation (COF1-COF2) $8.0 \%(2 / 25)$ and only one brand of mono-formulated organophosphate was registered. However, $68.0 \%(17 / 25)$ of the commercial brands of acaricide registered were found to have been used in the study farms. Overall, amitraz accounted for $36.9 \%$ (48/130) of the total acaricide formulations used for tick control followed by COF 30.0 \% (39/130), SP 27.7 \% (36/ 130) and mono-formulated OP $5.4 \%(7 / 130)$ being the least used class of acaricide. Within the same molecule, clear brand preferences were recorded. For example two brands of amitraz (A3 and A4), four brands of SP (SP1, SP2, SP3 and SP13) and 1 brand of COF (COF1) were preferred by $75.0 \%(36 / 48), 69.4 \%(25 / 36)$ and $71.8 \%$ $(28 / 39)$ of the farmers, respectively. The majority of the farmers $(81.3 \%, n=48)$ used at least two classes of acaricides within the last 2 years. Acaricide registration pattern showed that the rapid influx of different acaricide brands began in 1997 and its climax was attained in 2007. Between 1997 and 1998, all the three classes of acaricides (amidine, SP and OP) were on the Ugandan market suggesting that they have been in use for over 16 years in Uganda (Table 2).

Table 2 Acaricide molecules registered in Uganda and report of their use by the farmers

\begin{tabular}{|c|c|c|c|c|c|c|c|c|}
\hline Classification & $\begin{array}{l}\text { Brand } \\
\text { names, } \\
\text { total } \\
\text { number } \\
\text { and \% } \\
\text { freq. }\end{array}$ & Generic name & $\begin{array}{l}\text { Dilution } \\
\text { (acaricide } \\
\text { (ml: water } \\
\text { (liters)) }\end{array}$ & $\begin{array}{l}\text { Concentration } \\
\text { (\%) }\end{array}$ & $\begin{array}{l}\text { Freq. of } \\
\text { use by } \\
\text { farmers } \\
\text { in study } \\
\text { area }\end{array}$ & $\begin{array}{l}\% \\
\text { within } \\
\text { class }\end{array}$ & $\begin{array}{l}\text { Overall } \\
\%\end{array}$ & $\begin{array}{l}\text { Year } \\
\text { licensed } \\
\text { by NDA }\end{array}$ \\
\hline \multirow[t]{7}{*}{ Amidine } & A1 & Amitraz & $2: 1$ & 12.5 & 1 & 2.1 & 0.8 & 2000 \\
\hline & $\mathrm{A} 2$ & Amitraz & $2: 1$ & 12.5 & 8 & 16.7 & 6.2 & 2001 \\
\hline & A3 & Amitraz & $2: 1$ & 12.5 & 25 & 52.1 & 19.2 & 1998 \\
\hline & A4 & Amitraz & $2: 1$ & 12.5 & 11 & 22.9 & 8.5 & 1997 \\
\hline & A5 & Amitraz & $2: 1$ & 12.5 & 3 & 6.3 & 2.3 & 1997 \\
\hline & A6 & Amitraz & $2: 1$ & 12.5 & 0 & 0.0 & 0.0 & 1998 \\
\hline & A7 & Amitraz & $2: 1$ & 12.5 & 0 & 0.0 & 0.0 & 2007 \\
\hline Sub-total & $7(28.0)$ & & & & 48 & 100.0 & 36.9 & \\
\hline \multirow[t]{15}{*}{ Synthetic Pyrethroid } & SP1 & a-Cypermethrin & $1: 1$ & 5.0 & 8 & 22.2 & 6.2 & 2002 \\
\hline & SP2 & a-Cypermethrin & $1: 1$ & 5.0 & 5 & 13.9 & 3.8 & 1998 \\
\hline & SP3 & a-Cypermethrin & $1: 2$ & 10.0 & 7 & 19.4 & 5.4 & 2009 \\
\hline & SP4 & a-Cypermethrin & $1: 1$ & 7.0 & 3 & 8.3 & 2.3 & 2011 \\
\hline & SP5 & Cypermethrin & $1: 1$ & 10.0 & 1 & 2.8 & 0.8 & 1998 \\
\hline & SP6 & Cypermethrin & $1: 1$ & 10.0 & 0 & 0.0 & 0.0 & 1998 \\
\hline & SP7 & Cypermethrin & $1: 1$ & 15.0 & 0 & 0.0 & 0.0 & 2007 \\
\hline & SP8 & Cypermethrin & $1: 1$ & 10.0 & 0 & 0.0 & 0.0 & 2005 \\
\hline & $S P 9^{a}$ & Deltamethrin & $1: 1$ & 5.0 & 2 & 5.6 & 1.5 & - \\
\hline & SP10 & Deltamethrin & $1: 1$ & 5.0 & 4 & 11.1 & 3.1 & 2007 \\
\hline & SP11 & Deltamethrin & $1: 1$ & 5.0 & 0 & 0.0 & 0.0 & - \\
\hline & $\mathrm{SP} 12^{\mathrm{b}}$ & Flumethrin & - & - & 0 & 0.0 & 0.0 & 1997 \\
\hline & SP13 & Flumethrin & $1: 1$ & 2.0 & 5 & 13.9 & 3.8 & 1997 \\
\hline & SP14 & Flumethrin & $1: 1$ & 2.0 & 0 & 0.0 & 0.0 & 2010 \\
\hline & SP15 & Cyhalothrin & $1: 1$ & 5.0 & 1 & 2.8 & 0.8 & 2013 \\
\hline Sub-total & $15(60.0)$ & & & & 36 & 100.0 & 27.7 & \\
\hline Organophosphate & OP (1(4)) & Chlorfenvinphos & $1: 2$ & 100 & 7 & 100.0 & 5.4 & 1997 \\
\hline \multirow[t]{2}{*}{ Co-formulation } & COF1 & Chlorfenvinphos + a-cypermethrin & $1: 2$ & $30: 3$ & 28 & 71.8 & 21.5 & 2004 \\
\hline & COF2 & Chlorpyriphos + Cypermethrin & $1: 2$ & $50: 5$ & 11 & 28.2 & 8.5 & 2013 \\
\hline Sub-total & $2(8.0)$ & & & & 39 & 100.0 & 30.0 & \\
\hline Total & $25(100)$ & & & & 130 & & 100 & \\
\hline
\end{tabular}

aderegistered, 'pour-on, (-) No information' 


\section{Strength variation of SP acaricides sold on the Ugandan market}

As shown in Table 2, all (100\%) of the amitraz brands available on the market had a concentration of $12.5 \%$ (wt/vol.). However, 13/15 of the brands of synthetic pyrethroids licensed as emulsified concentrates had concentrations ranging from 2 to $15 \%$. The $38.5 \%(5 / 13)$ of synthetic pyrethroid brands were $5 \%$ (wt/vol.) followed by $10 \%$ wt.vol (4/13), $2 \%$ wt/vol. (2/13), $7 \%$ wt/vol $(1 / 13)$ and $15 \%$ wt/vol (1/15). Moreover, aside from one molecule, the rest were prescribed in a dilution ratio of acaricide to water of $1 \mathrm{ml}$ : 1liter, giving a wide concentration range for chemical tick control in Uganda. Similarly, the two co-formulations on the market had a wide concentration range despite the same dilution ratio of acaricide to water of 1:2 (Table 2).

\section{Susceptibility of tick larvae against the various molecules used}

The percentage mortality of larvae against the different acaricides used in the bioassay at DD and $2 \times \mathrm{DD}$ is shown in Table 3. Based on the WHO criteria, $93.5 \%$ $(29 / 31)$ of the tick populations tested had resistance to at least one class of acaricide molecule. Acaricide resistance was detected in Rhipicephalus ticks only.

\section{Resistance to synthetic pyrethroids}

At DD, $90.0 \%(27 / 30)$ of the ticks tested were resistant to both cypermethrin and deltamethrin. Doubling the concentration $(2 \times \mathrm{DD})$ of both chemicals did not cause any significant increase in mortality of the above ticks since $86.7 \%(26 / 30)$ remained resistant (Fig. 1). Moreover at $2 \times \mathrm{DD}, 60.0 \%(18 / 30)$ and $63.3 \%(19 / 30)$ were super resistant ( $0 \%$ mortality) against cypermethrin and deltamethrin, respectively. Of major concern was the fact that the $R$. appendiculatus collected from cattle in Gulu abattoir (northern region) was among the super resistant ticks (Table 3). Information gathered from the abattoir indicated that cattle from which the $R$. appendiculatus ticks were collected had originated from central Uganda. On the other hand, both A.variegatum from Gulu and $H$. leachi from Kiruhura districts were $100 \%$ susceptible at DD for cypermethrin and deltamethrin.

\section{Resistance to organophosphate}

Mono-formulated OP (chlorfenvinphos) at DD was efficacious in $86.7 \%(26 / 30)$ of tick populations screened. However, $13.3 \%(4 / 30)$ of the one host tick $R$. decoloratus were resistant to DD of chlorfenvinphos (Fig. 1). The four tick populations that were resistant were collected from Wakiso, Mbarara and Kiruhura districts.

\section{Resistance to co-formulation}

At DD of co-formulation, resistance was detected in $43.3 \%(13 / 30)$ of the tick populations tested. Interestingly, even at $2 \times \mathrm{DD}$, the co-formulated acaricide could not provide the level of effectiveness that was shown by mono-formulated chlorfenvinphos at DD since $23 \%$ (7/ 30) tick populations tested remained resistant (Fig. 1). Of the 13 Rhipicephalus tick populations that were resistant to co-formulation, $76.9 \%(10 / 13)$ were $R$. decoloratus.

\section{Resistance to amitraz}

At the DD only $12.9 \%$ (4/31) of the tick populations tested had amitraz resistant Rhipicephalus ticks with mortalities ranging from 15.4 to $68.1 \%$. However, increasing the dose of amitraz to $2 \times \mathrm{DD}$ did not result into commensurate level of mortality. Three of the amitraz resistant tick populations were $R$. decoloratus from the greater Bushenyi area (Bushenyi and Mitoma district). One amitraz resistant $R$. appendiculatus tick population was from a farm in Rukungiri district (Table 3). In the current study, amitraz resistance was only recorded in the western part of Uganda.

\section{Multi-acaricide resistance by Rhipicephalus ticks}

The presence of single or multiple acaricide resistance in the study area is shown in Fig. 2. Resistance to single and multi-acaricide molecules was detected in $48.2 \%$ $(13 / 29)$ and $55.2 \%(16 / 29)$ of tick populations from farms with acaricide resistance, respectively. Of the multi-acaricide resistant Rhipicephalus ticks, $75 \%$ (12/ 16 ) were $R$. decoloratus and the rest were $R$. appendiculatus. Further statistical analysis revealed significant statistical difference $(p<0.05)$ in the occurrence of multiacaricide resistance between the two species of ticks. All the farms that used either SP and co-formulation or SP, OP and COF within the last 2 years had $100 \%(14 / 14)$ multi-acaricide resistant ticks. There was significant association between use of both SP and COF with resistance to two classes $(p<0.001)$. Kiruhura district had $100 \%(4 / 4)$ multi-acaricide resistant tick populations, followed by Mbarara (75 \%; 3/4) in the western Uganda. Ticks from the two farms in Wakiso district (central Uganda) were also multi-acaricide resistant. Farms that rotated acaricides wrongly had the highest cases of both single and multi-acaricide resistance.

\section{Farm practices aimed at mitigating acaricide failure}

To overcome acaricide failure, various coping strategies have been adopted by farmers although they were considered to potentially worsen the existing tick challenge. Buying different brand(s) of acaricide with little or no regard to similarity in active molecules with previous brand(s) used on the same farm was encountered. In 
Table 3 Percentage mortality of larvae against various classes of acaricides determined with LPT

\begin{tabular}{|c|c|c|c|c|c|c|c|c|c|c|c|c|}
\hline \multirow[t]{3}{*}{ District } & \multirow[t]{3}{*}{ Farm/Pop. ID } & \multirow[t]{3}{*}{ Tick species } & \multicolumn{10}{|c|}{$\%$ Mortality (Mean \pm SEM) } \\
\hline & & & \multicolumn{2}{|c|}{ Amitraz (mg/ml) } & \multicolumn{2}{|c|}{ Cypermethrin (mg/ml) } & \multicolumn{2}{|c|}{ Deltamethrin (mg/ml) } & \multicolumn{2}{|c|}{ Chlorfenvinphos (mg/ml) } & \multicolumn{2}{|c|}{ Chlorfenvinphos/cypermethrin (COF) (mg/ml) } \\
\hline & & & 0.25 & 0.5 & 0.05 & 0.1 & 0.05 & 0.1 & 0.5 & 1.0 & $0.3 / 0.03$ & $0.6 / 0.06$ \\
\hline Kampala & $\mathrm{C} 1$ & R. app. & $100 \pm 0.0$ & $100 \pm 0.0$ & 0 & 0 & 0 & 0 & $100 \pm 0.0$ & $100 \pm 0.0$ & $100 \pm 0.0$ & $100 \pm 0.0$ \\
\hline \multirow[t]{2}{*}{ Wakiso } & C2 & R. app. & $100 \pm 0.0$ & $100 \pm 0.0$ & 0 & 0 & 0 & 0 & $100 \pm 0.0$ & $100 \pm 0.0$ & $14.7 \pm 0.4$ & $100 \pm 0.0$ \\
\hline & C3 & B. decol. & $100 \pm 0.0$ & $100 \pm 0.0$ & 0 & 0 & 0 & 0 & $67.5 \pm 0.5$ & $94.5 \pm 0.5$ & $21.0 \pm 5.0$ & $39.0 \pm 3.0$ \\
\hline Mubende & C4 & B. decol. & $100 \pm 0.0$ & $100 \pm 0.0$ & 0 & 0 & 0 & 0 & $100 \pm 0.0$ & $100 \pm 0.0$ & $70.4 \pm 0.7$ & $93.7 \pm 0.8$ \\
\hline Mpigi & C5 & R. app. & $100 \pm 0.0$ & $100 \pm 0.0$ & $11.0 \pm 0.0$ & $11.5 \pm 0.5$ & 0 & $12.5 \pm 2.5$ & $82.5 \pm 5.5$ & $100 \pm 0.0$ & $79.0 \pm 1$ & $87.5 \pm 2.5$ \\
\hline Kiboga & C6 & R. app. & $100 \pm 0.0$ & $100 \pm 0.0$ & 0 & 0 & 0 & 0 & $100 \pm 0.0$ & $100 \pm 0.0$ & $98.85 \pm 1.2$ & $100 \pm 0.0$ \\
\hline \multirow[t]{2}{*}{ Gulu } & N1 & R. app. & $100 \pm 0.0$ & $100 \pm 0.0$ & 0 & 0 & 0 & 0 & $100 \pm 0.0$ & $100 \pm 0.0$ & $100 \pm 0.0$ & $100 \pm 0.0$ \\
\hline & N2 & A. vari. & $100 \pm 0.0$ & $100 \pm 0.0$ & $100 \pm 0.0$ & $100 \pm 0.0$ & $100 \pm 0.0$ & $100 \pm 0.0$ & $100 \pm 0.0$ & $100 \pm 0.0$ & $100 \pm 0.0$ & $100 \pm 0.0$ \\
\hline \multirow[t]{4}{*}{ Mbarara } & W1 & R. app. & $100 \pm 0.0$ & $100 \pm 0.0$ & 0 & 0 & 0 & 0 & $100 \pm 0.0$ & $100 \pm 0.0$ & $100 \pm 0.0$ & $100 \pm 0.0$ \\
\hline & W2 & B. decol. & $100 \pm 0.0$ & $100 \pm 0.0$ & 0 & 0 & 0 & 0 & $50.6 \pm 2.7$ & $92.5 \pm 0.6$ & 0 & $19.6 \pm 0.4$ \\
\hline & W3 & R. app. & $100 \pm 0.0$ & $100 \pm 0.0$ & 0 & 0 & 0 & 0 & $100 \pm 0.0$ & $100 \pm 0.0$ & $71.0 \pm 0.0$ & $92.0 \pm 0.5$ \\
\hline & W4 & R. app. & $100 \pm 0.0$ & $100 \pm 0.0$ & $6.8 \pm 1.1$ & $36.6 \pm 7$ & 0 & $46.5 \pm 11.5$ & $100 \pm 0.0$ & $100 \pm 0.0$ & $93.95 \pm 0.9$ & $100 \pm 0.0$ \\
\hline \multirow[t]{5}{*}{ Kiruhura } & W5 & B. decol. & $100 \pm 0.0$ & $100 \pm 0.0$ & 0 & 0 & 0 & 0 & $68.7 \pm 3.8$ & $76.50 \pm 9.8$ & $25.05 \pm 0.6$ & $57.1 \pm 11.3$ \\
\hline & W6 & B. decol. & $100 \pm 0.0$ & $100 \pm 0.0$ & 0 & 0 & 0 & $19.7 \pm 10.3$ & $74.45 \pm 3.9$ & $91.2 \pm 4.0$ & $62.7 \pm 5.7$ & $93.3 \pm 2.9$ \\
\hline & W7 & H. leach. & $100 \pm 0.0$ & $100 \pm 0.0$ & $100 \pm 0.0$ & $100 \pm 0.0$ & $100 \pm 0.0$ & $100 \pm 0.0$ & $100 \pm 0.0$ & $100 \pm 0.0$ & $100 \pm 0.0$ & $100 \pm 0.0$ \\
\hline & W8 & B. decol. & $100 \pm 0.0$ & $100 \pm 0.0$ & 0 & 0 & 0 & 0 & $100 \pm 0.0$ & $100 \pm 0.0$ & $66.5 \pm 2.2$ & $76.3 \pm 0.7$ \\
\hline & W9 & B. decol. & $100 \pm 0.0$ & $100 \pm 0.0$ & 0 & 0 & 0 & 0 & $100 \pm 0.0$ & $100 \pm 0.0$ & $56.0 \pm 1$ & $65 \pm 2.5$ \\
\hline \multirow[t]{2}{*}{ Bushenyi } & W10 & B. decol. & $68.1 \pm 1.9$ & $74.5 \pm 1.5$ & 0 & $10.8 \pm 1.5$ & 0 & $16.3 \pm 2.5$ & $92 \pm 0.5$ & $96.7 \pm 0.9$ & $96.2 \pm 1.6$ & $98.8 \pm 0$ \\
\hline & W11 & B. decol. & $100 \pm 0$ & $100 \pm 0.0$ & 0 & 0 & 0 & 0 & $80.2 \pm 0.15$ & $100 \pm 0.0$ & $49.3 \pm 2.7$ & $60.0 \pm 1.6$ \\
\hline \multirow[t]{3}{*}{ Mitoma } & W12 & B. decol. & $100 \pm 0$ & $100 \pm 0$ & $5.0 \pm 0.0$ & $13.5 \pm 1.5$ & $8.0 \pm 3.0$ & $15.5 \pm .5$ & $100 \pm 0$ & $100 \pm 0$ & $53.5 \pm 0.5$ & $100 \pm 0$ \\
\hline & W13 & B. decol. & $41.5 \pm 0.5$ & $62.5 \pm 1.5$ & $65.5 \pm 1.5$ & $72.0 \pm 2.0$ & $73.5 \pm 1.5$ & $91.5 \pm 2.5$ & $100 \pm 0.0$ & $100 \pm 0.0$ & $100 \pm 0.0$ & $100 \pm 0.0$ \\
\hline & W14 & B. decol. & $45.0 \pm 1.0$ & NT & NT & NT & NT & NT & NT & NT & NT & NT \\
\hline Sheema & W15 & B. decol. & $100 \pm 0.0$ & $100 \pm 0.0$ & 0 & 0 & 0 & 0 & $100 \pm 0.0$ & $100 \pm 0.0$ & $56.7 \pm 0.9$ & $62.7 \pm 2.4$ \\
\hline \multirow[t]{4}{*}{ Rukungiri } & W16 & B. decol. & $100 \pm 0.0$ & $100 \pm 0.0$ & 0 & 0 & 0 & 0 & $100 \pm 0.0$ & $100 \pm 0.0$ & $95.8 \pm 0.3$ & $100 \pm 0.0$ \\
\hline & W17 & R. app. & $100 \pm 0.0$ & $100 \pm 0.0$ & 0 & 0 & 0 & 0 & $100 \pm 0.0$ & $100 \pm 0.0$ & $100 \pm 0.0$ & $100 \pm 0.0$ \\
\hline & W18 & R. app. & $15.4 \pm 0.1$ & $16.7 \pm 1.3$ & $97.7 \pm 0.5$ & $100 \pm 0.0$ & $98.3 \pm 0.1$ & $100 \pm 0.0$ & $100 \pm 0.0$ & $100 \pm 0.0$ & $100 \pm 0.0$ & $100 \pm 0.0$ \\
\hline & W19 & R. app. & $100 \pm 0.0$ & $100 \pm 0.0$ & $10.7 \pm 0.4$ & $23.2 \pm 1.9$ & $12.0 \pm 1.3$ & $27.9 \pm 0.7$ & $100 \pm 0.0$ & $100 \pm 0.0$ & $100 \pm 0.0$ & $100 \pm 0.0$ \\
\hline \multirow[t]{3}{*}{ Sembabule } & W20 & B. decol. & $100 \pm 0.0$ & $100 \pm 0.0$ & $15.0 \pm 1.0$ & $26.0 \pm 3.0$ & $15.5 \pm 0.5$ & $24.0 \pm 2.0$ & $100 \pm 0.0$ & $100 \pm 0.0$ & $88.5 \pm 0.5$ & $95.0 \pm 0.0$ \\
\hline & W21 & R. app. & $100 \pm 0.0$ & $100 \pm 0.0$ & 0 & 0 & 0 & 0 & $100 \pm 0.0$ & $100 \pm 0.0$ & $100 \pm 0.0$ & $100 \pm 0.0$ \\
\hline & W22 & R. app. & $100 \pm 0.0$ & $100 \pm 0.0$ & 0 & 0 & 0 & 0 & $100 \pm 0.0$ & $100 \pm 0.0$ & $100 \pm 0.0$ & $100 \pm 0.0$ \\
\hline Serere & E1 & B. decol. & $100 \pm 0.0$ & $100 \pm 0.0$ & $79.2 \pm 4.7$ & $91.7 \pm 1.1$ & $78.7 \pm 6.1$ & $95.0 \pm 1.9$ & $100 \pm 0.0$ & $100 \pm 0.0$ & $100 \pm 0.0$ & $100 \pm 0.0$ \\
\hline
\end{tabular}

R. app. (Rhipicephalus appendiculatus); B. decol. (Rhipicephalus (Boophilus) decoloratus); A. vari. (Amblyoma variegatum); H.leach (Haemaphysalis leachi);COF, coformulation; NT not tested due to few larvae, Pop Tick population; N1 and N2 are two tick population collected from abattoir (designated as "one farm" for purpose of this study) 
Susceptible ( $>80 \%$ mortality) Resistant $(<80 \%$ mortality)

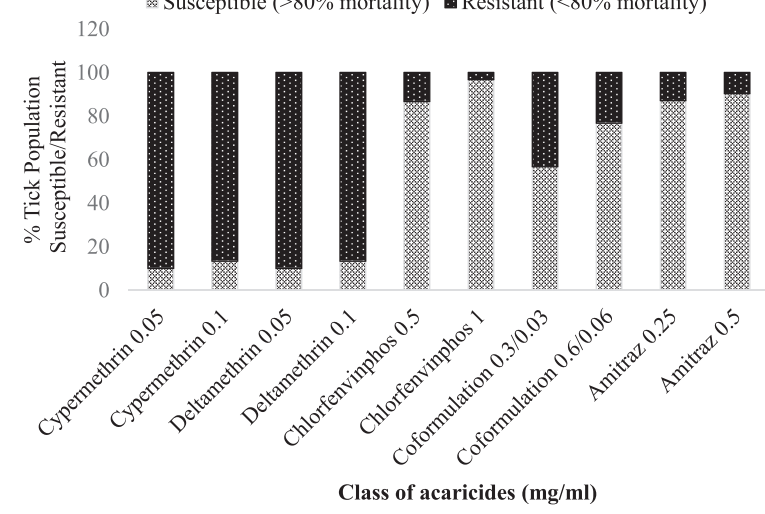

Fig. 1 Tick resistance status against various classes of acaricides. Thirty-one tick populations from 31 farms were tested for determining amitraz resistance. Tick resistance to SP, OP and COF were determined using 30 tick populations from 30 farms

two years, $74.1 \%(20 / 27)$ of the farms whose tick acaricide resistance status was determined used two to three acaricide molecules, and $55.6 \%(15 / 27)$ rotated the molecules wrongly. Rotation within the same molecule through purchase of different brands was recorded in $40.7 \%(11 / 27)$ of the farms. In addition, $25.9 \%(7 / 27)$ of the farmers increased the concentration of acaricide at least twice over the recommended strength. Some $14.8 \%(4 / 27)$ of the farmers shortened acaricide application interval to twice a week (every three days). This translates into approximately 10 exposures every month and 120 exposures annually. Mixing of two different acaricide formulations was encountered in $7 \%(2 / 27)$ of farms and one of the farms mixed co-formulation and amitraz, thus exposing ticks to all the three molecules at once. In a farm that mixed two acaricides and sprayed twice every week, damage to the skin of cattle due to frequent spraying with higher acaricide strength was encountered. As a result, the ticks were easily picked with the damaged skin (Additional file 2: Figure S2).

\section{Discussion}

This is the first report that has comprehensively investigated tick acaricide resistance since the introduction of synthetic pyrethroid, co-formulations and amitraz in Uganda. Rhipicephalus ticks are widespread in the country [4], posing a serious threat especially to exotic cattle. Thus TBD especially ECF is ranked by farmers as the most important constraint to cattle production in Uganda [28, 29]. Acaricides are therefore perceived as the most efficient way of controlling ticks and preventing the above diseases. However, with over 25 brands of all the major classes of acaricides circulating on the market (Table 2), farmers are "spoiled for choice". SP and amitraz accounted for $88 \%$ of the total acaricide brands marketed although amitraz was the most preferred by farmers during the study. This finding is consistent with what was previously reported in north eastern Uganda [30]. Of concern was the variation in strength of the different SP whose dilutions are similar, thus giving different concentrations. It may be possible that amongst cypermethrin, variation in strength may reflect the proprietary difference in composition of cis and trans isomers. However, there is need for regulatory harmonization of strength of SP formulations with similar active ingredients, notwithstanding inappropriate application practices by farmers. A noticeable example of inappropriate acaricide use was wrong rotation of acaricides between molecules and rotation of acaricides within the same molecule under different brand names. It was also widely believed by farmers that acaricide failure could only be caused by "fake" chemicals. This clearly indicates that farmers lacked knowledge on possibility of ticks becoming resistant to chemicals due irrational acaricide use.

In this study, $93.5 \%(29 / 31)$ of the larval population tested had resistance to at least one class of acaricide molecule; all of them belonging to the genus Rhipicephalus. In Uganda, acaricide resistance was first diagnosed in Rhipicephalus ticks against organochlorine, toxaphen in 1970s [11]. This occurred mainly due to increased acaricide pressure considering a compulsory tick control committee enforced weekly dipping of cattle across the country. However, subsequent zoning of acaricides and restricting circulation to the district veterinary office were reported as efficient strategies in delaying acaricide resistance. Nevertheless, political strife in early 1970s [6, 31] and further liberalization of the veterinary drug sector [30] ended both zoning and control in supply of acaricides leading to widespread inappropriate acaricide use. Of major concern now is the high level of resistance to SP (90\%) and emergence of super resistant R. appendiculatus and $R$. decoloratus ticks in at least $60 \%$ of the tick 


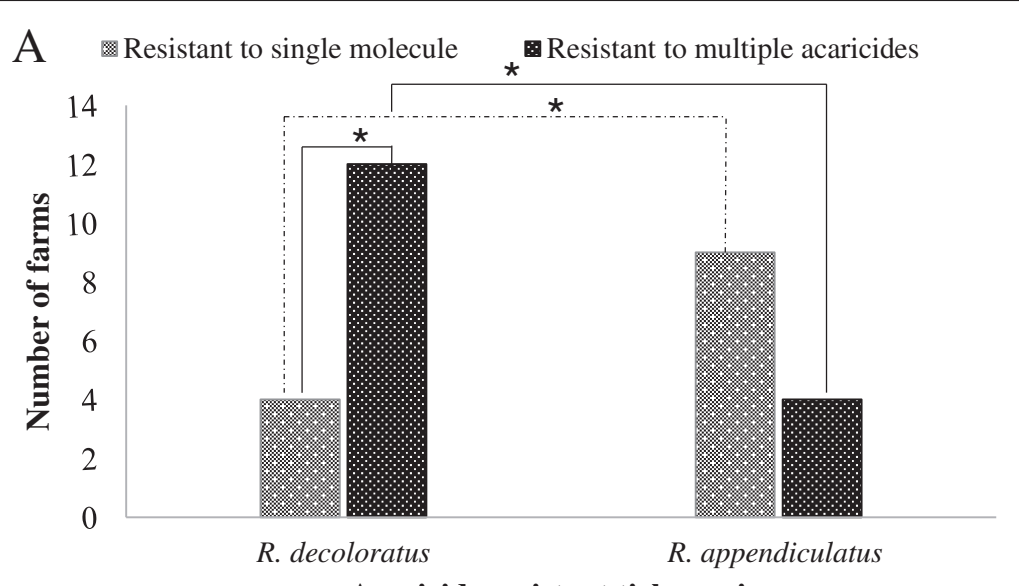

Acaricide resistant tick species
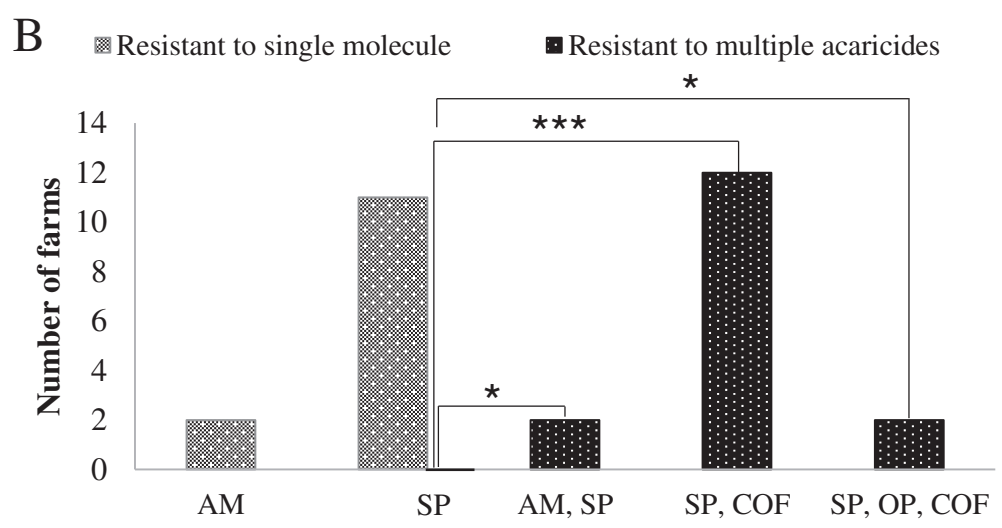

Class of acaricide used on the farm in the last 2 years

C Resistant to single molecule $\quad$ Resistant to multiple acaricides

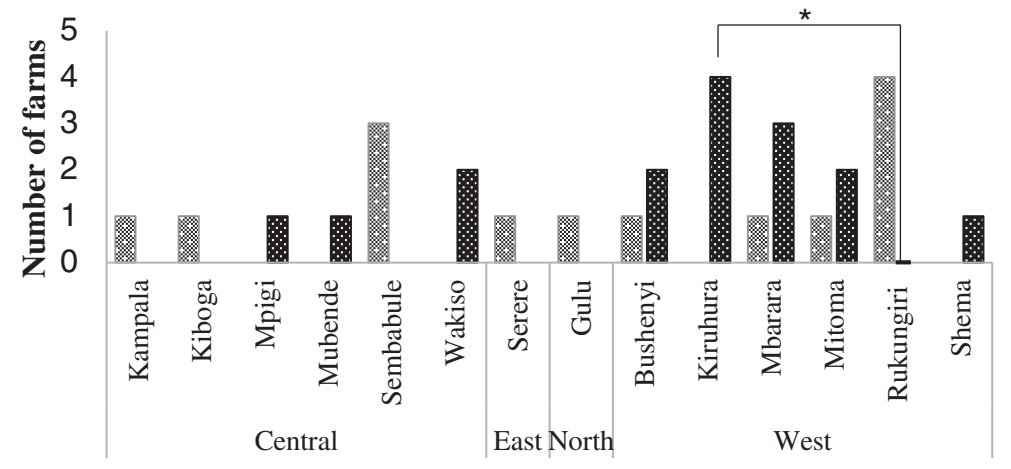

Region/ Districts

Fig. 2 (See legend on next page.) 


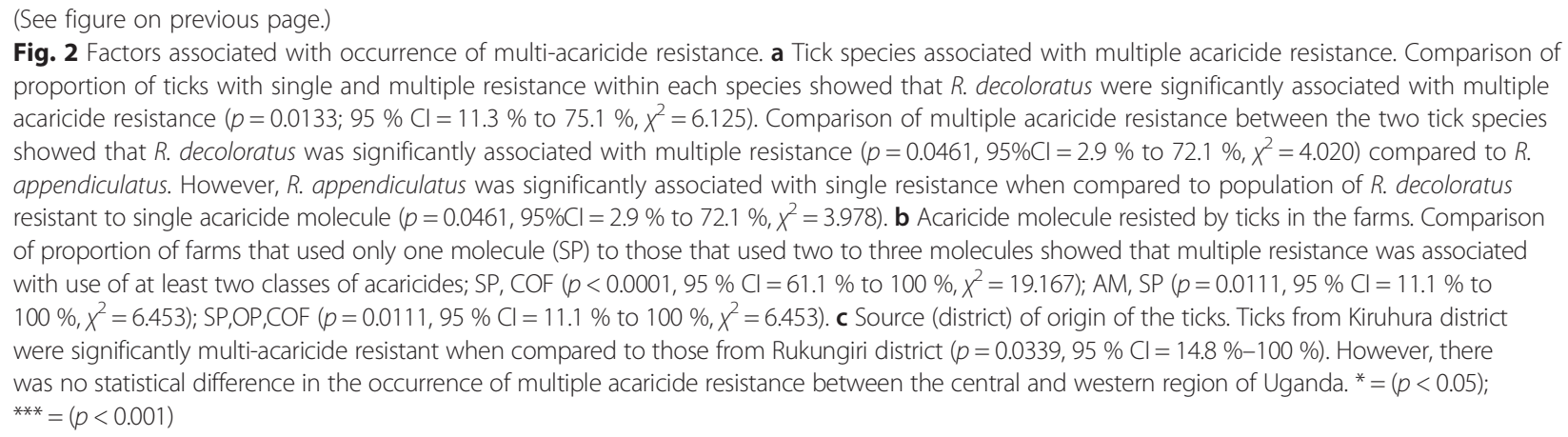

populations investigated in this study (Table 3). Since their introduction, SP have enjoyed unique preference due to their dual effect against both ticks and flies [30]. However, its irrational use for over 16 years especially by farmers who use the spray method, could have selected for stable resistance. Studies carried out in related tick-R. microplus have attributed such level of resistance to multiple mutations in SP target site, voltage sensitive sodium channel domains II and III [18, 32-34] . A similar level of resistance was first observed in insects and attributed to knock down resistance $(k d r)$ in the sodium channel [35-39]. It should be noted that the prevalence of SP resistance by Rhipicephalus ticks (96.4\%) reported in this study is amongst the highest compared to those previously known in South America [40-42], India [43] and the rest of Africa [44-47]. Possible evidence of cross-resistance between SP and OP was also observed in $30 \%$ of the tick populations from farms that used co-formulated acaricides. Previous studies in cattle tick showed that ticks that were resistant to SP and OP had elevated esterase activity $[26,48]$. The apparent lack of synergism between SP and OP observed in this study possibly emanates from the fact that the most dominant co-formulation used in Uganda (COF1) is prescribed at 1.7 times lower concentration than their corresponding mono-formulations. While the pharmacological basis for such formula is justifiable under ideal conditions, its efficacy is bound to be low in a situation where resistance has emerged against one of the chemicals. This eventually could act as a recipe for emergence of resistance against what otherwise would be the effective molecule (OP) in the co-formulation due to suboptimal exposure dose. This possibly explains the low efficacy recorded against OP in farms with SP resistant ticks that were also previously exposed to co-formulated acaricides. The mono-formulated OP chlorfenvinphos showed promising efficacy, partly because it is not widely used. The low farm use may be attributed to factors such as shorter application interval recommended for its use and low margin of safety compared to other classes of acaricides. However, emergence of resistance against co-formulation containing OP is an early indication that resistance to this group of acaricides is progressively building amidst fear of possible cross-resistance with SP.

Amitraz resistance was the least detected (12.9 \%) in the current study thus corroborating with the findings on in its use at farm level. This finding is consistent with previous studies $[49,50]$. Although amitraz formulations have been the dominantly mentioned acaricides (36.9\%), their routine use has remained low due to their narrow spectrum of benefit compared to SP, as far as fly repellence is concerned. This explains why some farmers irrationally mixed amitraz and SP formulations. On the other hand, the increase in amitraz use may be an indicator that farmers were getting better tick control results with amitraz following negative experience while using SP and COF. However, the resistance observed against amitraz in $12.9 \%$ of the tick populations may be mediated by mutation in the amitraz target, octopamine receptor [51-53]. Nevertheless, the high level of multiacaricide resistance $(55.2 \%)$ and emergence of isolated amitraz resistance ticks further emphasizes the need for accelerated intervention to combat their spread across the country. The super SP resistant $R$. appendiculatus collected in Gulu abattoir from cattle bought from central Uganda should be an example of how such ticks can be easily spread through cattle trade and/or movement. Therefore, creation of farm awareness, vigilance amongst veterinarians and cattle traders, and promoting use of amidines in farming communities with ticks that are resistant to SP and coformulation could potentially lead to containment of resistant tick populations. However, the use of amitraz should factor into account the balance between need for tick and tsetse fly control, especially in areas that are known to be tsetse infested as previously reported [30]. In the absence of technical intervention, coping strategies employed by farmers experiencing acaricide failure are likely to worsen the existing challenge. This includes exponential rise in irrational admixing of various acaricide formulations into cocktail and short application intervals that will cause collateral 
damage to cattle (Additional file 2: Figure S2), food safety and public health. Although alternative technologies such as vaccination of cattle with Muguga cocktail ECF vaccine is being promoted and said to be effective against ECF [54], the emergence of acaricide resistant $R$. decoloratus undermines such efforts. Without controlling the above ticks, babesiosis and anaplasmosis will certainly cause economic losses despite immunization against ECF. Therefore, there is need for various actors in the animal industry to jointly identify strategies for mitigation of acaricide resistance in Uganda. This, however, requires close collaboration between the various stakeholders in the acaricide supply chain and research animal health institutions in the country [55].

\section{Conclusion}

This research is the first in Uganda to report emergence of super SP resistant and multi-acaricide resistant $R$. appendiculatus and $R$. decoloratus ticks. Our results further highlight the importance of routine monitoring of tick acaricide resistance for early detection and intervention especially in countries where veterinary drugs/acaricides are liberalized. In absence of technical interventions, farmer-led solutions aimed at troubleshooting for efficacy of the multitude of acaricides at their disposal are expected to potentially cause negative collateral effect on future chemical tick control options, animal welfare and public health. While understanding the molecular basis of such resistance and countrywide epidemiological studies are necessary, a multi-faceted approach directed towards containment and eradication of acaricide resistant ticks is urgently needed in Uganda.

\section{Additional files}

Additional file 1: Figure S1. Map of Uganda showing the various districts from which tick samples were collected. A, Map of Africa showing Uganda. B, Map of Uganda showing the areas from which ticks were collected (depicted by ticks). (PPTX $656 \mathrm{~kb}$ )

Additional file 2: Figure S2. $R$. decoloratus picked from cattle with acaricide induced skin damage. A, Hair bundle (h) that detached from the skin of cattle as the tick ( $\mathrm{t}$ ) was picked; B, Damaged cattle skin (s) that was easily detached with the tick ( $\mathrm{t})$; C, The piece of damaged skin (s) firmly attached to the mouth part thus altering the gross morphological appearance of the cephalus region of tick (t); farmer considered these "new" species of ticks. (PPTX $406 \mathrm{~kb}$ )

\section{Competing interests}

The authors declare that they have no competing interests.

\section{Authors' contributions}

Conceived and designed the study: PV, KF, JOA, XX, RT, MK, HS. Collected ticks samples from the field: PV, DT, JB, JBM, SK. Rearing of experimental ticks in the laboratory: PV, MK, KF, JB, SK. Taxonomic identification of ticks: KF, NT, RUS, TH, PV. Performed chemical tick assays: PV, EW, JB, RO, DT, KF, DOO. Wrote the draft manuscript: PV, RUS, XX, EW, RT, JOA, KF, HS. All the authors read and approved the final manuscript submitted.

\section{Acknowledgements}

This research was supported by Japan International Cooperation (JICA). We thank the National Drug Authority for donating reference standards and the Acaricide Testing Laboratory of Ministry of Agriculture, Animal Industry and Fisheries for analysis of the commercial acaricides used. We also appreciate the contribution of Dr. Margaret Saimo-Kahwa, the staff of CDL, Pharmaceutical and Toxicology Research Laboratory during the study. The contribution of Dr. Nabi Oki, Mr. Dickson Ndoboli, Dr. Charles Lagu, Dr. Simon Peter Musinguzi, Dr. William Mwebembezi, Dr. Toni Aliro, Dr. Rogers Sebyoto, Dr .Keneth I. Kasozi, Dr. Isaac Kikozza, and Dr. Joseph Ruhinda in sample collection are highly appreciated. The contributions of Dr. Benedicto Byamukama, Mr. Johnson Bbira and Ms. Mariam Komugisha in establishment of initial in-house procedure for tick assay and guidance given by Dr. Charoonluk Jirapattharasate in data analysis are appreciated.

\section{Author details}

'Department of Veterinary Pharmacy, Clinics and Comparative Medicine, School of Veterinary Medicine and Animal Resources, College of Veterinary Medicine, Animal Resources and Biosecurity, Makerere University, P. O Box 7062, Kampala, Uganda. ${ }^{2}$ Central Diagnostic Laboratory, College of Veterinary Medicine, Animal Resources and Biosecurity, Makerere University, P. O Box 7062, Kampala, Uganda. ${ }^{3}$ National Research Center for Protozoan Diseases, Obihiro University of Agriculture and Veterinary Medicine, Inada-Cho, Obihiro, Hokkaido 080-8555, Japan. ${ }^{4}$ Drug Information Department, National Drug Authority, P. O. Box 23096, Kampala, Uganda. ${ }^{5}$ Directorate of Research and Graduate Training, Makerere University, P.O Box 7062, Kampala, Uganda. ${ }^{6}$ Laboratory of Parasitic Diseases, National Institute of Animal Health, National Agricultural and Food Research Organization, 3-1-5 Kannondai, Tsukuba, Ibaraki 305-0856, Japan. 'Present Address: Department of Parasitology, Kitasato University School of Medicine, Kitasato, Minami-ku, Sagamihara, Kanagawa 252-0374, Japan. ${ }^{8}$ Present Address: Japan International Cooperation Agency, Nibancho Center Building, 5-25, Nibancho, Chiyoda-ku, Tokyo 108-8012, Japan.

Received: 27 August 2015 Accepted: 21 December 2015 Published online: 04 January 2016

References

1. Mukhebi AW, Perry BD, Kruska R. Estimated economics of theileriosis control in Africa. Prev Vet Med. 1992;12:73-85.

2. Norval RAl, Perry BD, Young AS. The epidemiology of theileriosis in Africa. London, UK: Academic Press; 1992. 481

3. Minjauw B, McLeod A. Tick-borne diseases and poverty. The impact of ticks and tickborne diseases on the livelihood of small-scale and marginal livestock owners in India and eastern and southern Africa, DFID Animal Health Programme. Edinburgh: Centre for Tropical Veterinary Medicine, University of Edinburgh, Programme DAH; 2003.

4. Otim CP. Theileriosis in Uganda. In: Dolan T, editor. Theileriosis in eastern, central and southern Africa, Proceedings of a Workshop on East Coast Fever Immunization, Held in Lilongwe, Malawi, 20-2 September 1988. Nairobi, Kenya: The International Laboratory for Research on Animal Diseases; 1989.

5. Ocaido M, Muwazi RT, Opuda JA. Economic impact of ticks and tick-borne diseases on cattle production systems around Lake Mburo National Park in south western Uganda. Trop Anim Health Prod. 2009;41:731-9.

6. Okello-Onen J, Ssekitto CMB, Ssentongo YK, Kudamba CAL. Tick situation and control strategies in Uganda. Int J Trop Insect Sci. 1992;13(Special Issue 04):657-60.

7. Mugisha A, McLeod A, Percy R, Kyewalabye E. Strategies, effectiveness and rationale of vector-borne disease control in the pastoralist system of south-western Uganda. Trop Anim Health Prod. 2005;37:479-89.

8. Mugabi KN, Mugisha A, Ocaido M. Socio-economic factors influencing the use of acaricides on livestock: a case study of the pastoralist communities of Nakasongola district, central Uganda. Trop Anim Health Prod. 2010;42:131-6.

9. Aguilar-Tipacamu G, Rosario-Cruz R, Miller RJ, Guerrero FD, Rodriguez-Vivas Rl, Garcia-Vazquez Z. Phenotype changes inherited by crossing pyrethroid susceptible and resistant genotypes from the cattle tick Riphicephalus (Boophilus) microplus. Exp Appl Acarol. 2011;54:301-11.

10. Abbas RZ, Zaman MA, Colwell DD, Gilleard J, lqbal Z. Acaricide resistance in cattle ticks and approaches to its management: the state of play. Vet Parasitol. 2014;203:6-20. 
11. Kitaka FX, Oteng AK, Kamya EP. Toxaphene-resistant ticks occurring on cattle in Uganda: Boophilus decolaratus, Rhipicephalus evertsi and Rhipicephalus appendiculatus. Bull Epizoot Dis Afr. 1970;18:137-42.

12. Foil LD, Coleman P, Eisler M, Fragoso-Sanchez H, Garcia-Vazquez Z, Guerrero $\mathrm{FD}$, et al. Factors that influence the prevalence of acaricide resistance and tick-borne diseases. Vet Parasitol. 2004;125:163-81.

13. Food and Agriculture Organization of the United Nation. Ticks and tickborne disease control: a practical field manual. Vol. 1. Food and Agriculture Organization of the United Nations. 1984.

14. Shaw RD. Culture of an organophosphorus-resistant strain of Boophilus microplus (Can.) and an assessment of its resistance spectrum. Bull Entomol Res. 1966;56:389-405.

15. Drummond RO, Ernst SE, Trevino JL, Gladney WJ, Graham OH. Boophilus annulatus and B. microplus: laboratory tests of insecticides. J Econ Entomol. 1973;66:130-3.

16. Lovis L, Guerrero FD, Miller RJ, Bodine DM, Betschart B, Sager H. Distribution patterns of three sodium channel mutations associated with pyrethroid resistance in Rhipicephalus (Boophilus) microplus populations from north and south America, South Africa and Australia. Int J Parasitol Drugs Drug Resist. 2012;2:216-24

17. Guerrero FD, Davey RB, Miller RJ. Use of an allele-specific polymerase chain reaction assay to genotype pyrethroid resistant strains of Boophilus microplus (Acari: Ixodidae). J Med Entomol. 2001;38:44-50.

18. Morgan JA, Corley SW, Jackson LA, Lew-Tabor AE, Moolhuijzen PM, Jonsson NN. Identification of a mutation in the para-sodium channel gene of the cattle tick Rhipicephalus (Boophilus) microplus associated with resistance to synthetic pyrethroid acaricides. Int J Parasitol. 2009;39:775-9.

19. Faza AP, Pinto IS, Fonseca I, Antunes GR, Monteiro CM, Daemon E, et al. A new approach to characterization of the resistance of populations of Rhipicephalus microplus (Acari: Ixodidae) to organophosphate and pyrethroid in the state of Minas Gerais, Brazil. Exp Parasitol. 2013;134:519-23.

20. Domingues LN, Bello ACPP, Cunha AP, Brasil BSAF, Barros ATM, Leite RC, et al. Survey of pyrethroid and organophosphate resistance in Brazilian field populations of Rhipicephalus (Boophilus) microplus: Detection of C190A mutation in domain II of the para-type sodium channel gene. Vet Parasitol. 2012;189:327-32.

21. Balikowa D. A Review of Uganda's Dairy Industry. Kampala: GOU/FAO Dairy Project, TCP/UGA/3202(D); Senior Business Advisor, TechnoServe/East Africa Dairy Development Project (EADD); 2011. http://www.fao.org/3/a-aq292e. pdf. [Accessed: 11/08/2015].

22. Walker AR, Bouattour A, Camicas J-L, Estrada-Peña A, Horak IG, Latif A, et al. Ticks of Domestic Animals in Africa: a Guide to Identification of Species. Ednburgh: The University of Edinburgh; 2003.

23. WHO. Test procedures for insecticide resistance monitoring in malaria vector mosquitoes. Geneva: World Health Organization; 2013.

24. FAO. Ticks Acaricide Resistance: Diagnosis, Management and Prevention. Rome: FAO; 2004

25. Miller RJ, Davey RB, White WH, George JE. A comparison of three bioassay techniques to determine amitraz susceptibility in Boophilus microplus (Acari: Ixodidae). J Med Entomol. 2007;44:283-94.

26. Hernandez R, He H, Chen AC, Waghela SD, Wayne Ivie G, George JE, et al. Identification of a point mutation in an esterase gene in different populations of the southern cattle tick, Boophilus microplus. Insect Biochem Mol Biol. 2000;30:969-77.

27. Rosario-Cruz R, Almazan C, Miller RJ, Dominguez-Garcia DI, Hernandez-Ortiz $\mathrm{R}$, de la Fuente J. Genetic basis and impact of tick acaricide resistance. Front Biosci (Landmark Ed). 2009;14:2657-65.

28. Muhanguzi D, Waiswa C, Matovu E. Prevalence and characterization of Theileria and Babesia species in cattle under different husbandry systems in Western Uganda. J Anim Vet Adv. 2010;2:51-8.

29. Kabi F, Masembe C, Muwanika V, Kirunda H, Negrini R. Geographic distribution of non-clinical Theileria parva infection among indigenous cattle populations in contrasting agro-ecological zones of Uganda: implications for control strategies. Parasit Vectors. 2014;7:414

30. Bardosh K, Waiswa C, Welburn SC. Conflict of interest: use of pyrethroids and amidines against tsetse and ticks in zoonotic sleeping sickness endemic areas of Uganda. Parasit Vectors. 2013;6:204.

31. Bwangamoi O. Theileriosis in Uganda. In: Immunization against Theileriosis in Africa; 1984; International Laboratory for Research on Animal Diseases,
Nairobi, 1989. https://www.ilri.org/InfoServ/Webpub/fulldocs/Immune71/ theugand.htm. [Accessedd: 11/08/2015].

32. Jonsson NN, Cutulle C, Corley SW, Seddon JM. Identification of a mutation in the para-sodium channel gene of the cattle tick Rhipicephalus microplus associated with resistance to flumethrin but not to cypermethrin. Int J Parasitol. 2010:40:1659-64.

33. Guerrero FD, Lovis L, Martins JR. Acaricide resistance mechanisms in Rhipicephalus (Boophilus) microplus. Rev Bras Parasitol Vet. 2012;21:1-6.

34. Stone NE, Olafson PU, Davey RB, Buckmeier G, Bodine D, Sidak-Loftis LC, et al. Multiple mutations in the para-sodium channel gene are associated with pyrethroid resistance in Rhipicephalus microplus from the United States and Mexico. Parasit Vectors. 2014;7:456.

35. Pittendrigh B, Reenan R, Ffrench-Constant RH, Ganetzky B. Point mutations in the Drosophila sodium channel gene para associated with resistance to DDT and pyrethroid insecticides. Mol Gen Genet. 1997:256:602-10.

36. Dong K, Valles SM, Scharf ME, Zeichner B, Bennett GW. The knockdown resistance $(k d r)$ mutations in pyrethroid-resistant German cockroaches. Pestic Biochem Physiol. 1998;60:195-204.

37. Soderlund DM, Knipple DC. The molecular biology of knockdown resistance to pyrethroid insecticides. Insect Biochem Mol Biol. 2003;33:563-77.

38. Tan WL, Li CX, Wang ZM, Liu MD, Dong YD, Feng XY, et al. First detection of multiple knockdown resistance $(k d r)$-like mutations in voltage-gated sodium channel using three new genotyping methods in Anopheles sinensis from Guangxi Province, China. J Med Entomol. 2012;49:1012-20.

39. Dong K, Du Y, Rinkevich F, Nomura Y, Xu P, Wang L, et al. Molecular biology of insect sodium channels and pyrethroid resistance. Insect Biochem Mol Biol. 2014;50:1-17.

40. Mendes MC, Lima CK, Nogueira AH, Yoshihara E, Chiebao DP, Gabriel $\mathrm{FH}$, et al. Resistance to cypermethrin, deltamethrin and chlorpyriphos in populations of Rhipicephalus (Boophilus) microplus (Acari: Ixodidae) from small farms of the State of Sao Paulo, Brazil. Vet Parasitol. 2011;178:383-8

41. Veiga LP, Souza AP, Bellato V, Sartor AA, Nunes AP, Cardoso HM. Resistance to cypermethrin and amitraz in Rhipicephalus (Boophilus) microplus on the Santa Catarina Plateau, Brazil. Rev Bras Parasitol Vet. 2012;21:133-6.

42. de Santana BB, Ramos RA, Santana Mde A, Alves LC, de Carvalho GA. Susceptibility of Rhipicephalus (Boophilus) microplus (Acari: Ixodidae) to pyrethroids and their associations in Pernambuco, Brazil. Rev Bras Parasitol Vet. 2013:22:276-80.

43. Sharma AK, Kumar R, Kumar S, Nagar G, Singh NK, Rawat SS, et al. Deltamethrin and cypermethrin resistance status of Rhipicephalus (Boophilus) microplus collected from six agro-climatic regions of India. Vet Parasitol. 2012:188:337-45.

44. Mekonnen S, Bryson NR, Fourie LJ, Peter RJ, Spickett AM, Taylor RJ, et al. Comparison of 3 tests to detect acaricide resistance in Boophilus decoloratus on dairy farms in the Eastern Cape Province, South Africa. J S Afr Vet Assoc. 2003;74:41-4.

45. Ntondini Z, van Dalen EM, Horak IG. The extent of acaricide resistance in 1-, 2- and 3-host ticks on communally grazed cattle in the eastern region of the Eastern Cape Province, South Africa. J S Afr Vet Assoc. 2008;79:130-5.

46. Lovis L, Perret JL, Bouvier J, Fellay JM, Kaminsky R, Betschart B, et al. A new in vitro test to evaluate the resistance level against acaricides of the cattle tick, Rhipicephalus (Boophilus) microplus. Vet Parasitol. 2011;182:269-80.

47. Adakal H, Stachurski F, Chevillon C. Tick control practices in Burkina Faso and acaricide resistance survey in Rhipicephalus (Boophilus) geigyi (Acari: Ixodidae). Exp Appl Acarol. 2013;59:483-91.

48. Rosario-Cruz R, Guerrero FD, Miller RJ, Rodriguez-Vivas RI, Tijerina M, Dominguez-Garcia Dl, et al. Molecular survey of pyrethroid resistance mechanisms in Mexican field populations of Rhipicephalus (Boophilus) microplus. Parasitol Res. 2009;105:1145-53.

49. Ducornez S, Barre N, Miller RJ, Garine-Wichatitsky M. Diagnosis of amitraz resistance in Boophilus microplus in New Caledonia with the modified Larval Packet Test. Vet Parasitol. 2005;130:285-92

50. Jonsson NN, Hope M. Progress in the epidemiology and diagnosis of amitraz resistance in the cattle tick Boophilus microplus. Vet Parasitol. 2007:146:193-8.

51. Chen AC, He H, Davey RB. Mutations in a putative octopamine receptor gene in amitraz-resistant cattle ticks. Vet Parasitol. 2007:148:379-83.

52. Baxter GD, Barker SC. Isolation of a CDNA for an octopamine-like, G-protein coupled receptor from the cattle tick, Boophilus microplus. Insect Biochem Mol Biol. 1999;29:461-7. 
53. Corley SW, Jonsson NN, Piper EK, Cutulle C, Stear MJ, Seddon JM. Mutation in the $R m B A O R$ gene is associated with amitraz resistance in the cattle tick Rhipicephalus microplus. Proc Natl Acad Sci U S A. 2013;110:16772-7.

54. Norling M, Bishop RP, Pelle R, Qi W, Henson S, Drábek EF, et al. The genomes of three stocks comprising the most widely utilized live sporozoite Theileria parva vaccine exhibit very different degrees and patterns of sequence divergence. BMC Genomics. 2015;16:729. doi:10.1186/ s12864-015-1910-9.

55. Graf JF, Gogolewski R, Leach-Bing N, Sabatini GA, Molento MB, Bordin EL, et al. Tick control: an industry point of view. Parasitology. 2004; 129(Suppl):S427-42

Submit your next manuscript to BioMed Central and we will help you at every step:

- We accept pre-submission inquiries

- Our selector tool helps you to find the most relevant journal

- We provide round the clock customer support

- Convenient online submission

- Thorough peer review

- Inclusion in PubMed and all major indexing services

- Maximum visibility for your research

Submit your manuscript at www.biomedcentral.com/submit
Biomed Central 